\title{
SINGULAR VECTORS ON MANIFOLDS AND FRACTALS
}

\author{
DMITRY KLEINBOCK, NIKOLAY MOSHCHEVITIN, AND BARAK WEISS
}

\begin{abstract}
We generalize Khintchine's method of constructing totally irrational singular vectors and linear forms. The main result of the paper shows existence of totally irrational vectors and linear forms with large uniform Diophantine exponents on certain subsets of $\mathbb{R}^{n}$, in particular on any analytic submanifold of $\mathbb{R}^{n}$ of dimension $\geq 2$ which is not contained in a proper rational affine subspace.
\end{abstract}

\section{INTRODUCTION}

1.1. Singular vectors and uniform Diophantine exponents. In this paper we consider uniform rational approximations to $n$-tuples of real numbers. Denote by $\langle x\rangle$ the distance from $x \in \mathbb{R}$ to the nearest integer, and for $\boldsymbol{x}=\left(x_{1}, \ldots, x_{n}\right) \in \mathbb{R}^{n}$ and $\boldsymbol{y}=\left(y_{1}, \ldots, y_{n}\right) \in \mathbb{R}^{n}$ let

$$
\langle\boldsymbol{x}\rangle \stackrel{\text { def }}{=}\left(\left\langle x_{1}\right\rangle, \ldots,\left\langle x_{n}\right\rangle\right), \quad\|\boldsymbol{x}\| \stackrel{\text { def }}{=} \max _{1 \leq j \leq n}\left|x_{j}\right|, \quad \boldsymbol{x} \cdot \boldsymbol{y} \stackrel{\text { def }}{=} x_{1} y_{1}+\cdots x_{n} y_{n} .
$$

A vector $\boldsymbol{\xi}=\left(\xi_{1}, \ldots, \xi_{n}\right)$ is called singular if for every $c>0$ the system of inequalities

$$
\|\langle q \boldsymbol{\xi}\rangle\| \leq c t^{-1 / n}, \quad 0<q \leq t
$$

has an integer solution $q$ for any sufficiently large $t$. Equivalently (in view of Khintchine's Transference Principle [19, 7]), $\boldsymbol{\xi}$ is singular if for every $c>0$ the system of inequalities

$$
\langle\boldsymbol{q} \cdot \boldsymbol{\xi}\rangle \leq c t^{-n}, \quad 0<\|\boldsymbol{q}\| \leq t
$$

has a solution $\boldsymbol{q} \in \mathbb{Z}^{n}$ for any sufficiently large $t$. We note that from Dirichlet's theorem, or, alternatively, from Minkowski's convex body theorem, it follows that when $c=1$, for all $t \geq 1$ both (1.1) and (1.2) have integer solutions. It is well-known that the set of singular real numbers coincides with $\mathbb{Q}$; thus in what follows we will assume that $n \geq 2$.

It was observed by Khintchine, see [7, Ch. V, §7] that singular vectors form a set of Lebesgue measure zero. One reason why singular vectors are an interesting object of study is their connection with homogeneous dynamics. It was showed by Dani [10] that $\xi$ is singular if and only if the trajectory of a certain lattice in $\mathbb{R}^{n+1}$ constructed from $\boldsymbol{\xi}$ diverges (i.e. leaves every compact subset of the space of lattices). We will not exploit this connection in the present paper; see however [25, 35, 11] for generalizations and further discussions. In particular, the Hausdorff dimension of the set of singular

Date: December 2019.

2010 Mathematics Subject Classification: Primary 11J13; Secondary 11J54, 37A17.

Key words and phrases: Diophantine approximation, singular vectors, uniform exponents, divergent trajectories. 
vectors in $\mathbb{R}^{n}$ was only relatively recently shown by Cheung and Chevallier [8] to be equal to $\frac{n^{2}}{n+1}$; see also an earlier work of Cheung [7] settling the case $n=2$.

One can also introduce different 'levels of singularity' of vectors $\boldsymbol{\xi} \in \mathbb{R}^{n}$ by considering exponents of uniform Diophantine approximation. Namely, one defines $\hat{\omega}(\boldsymbol{\xi})$, the uniform exponent of $\boldsymbol{\xi}$ in the sense of simultaneous approximation, as the supremum of $\gamma>0$ for which the system of inequalities

$$
\|\langle q \boldsymbol{\xi}\rangle\| \leq t^{-\gamma}, \quad 0<q \leq t
$$

has an integer solution $q$ for all $t$ large enough. Likewise, $\hat{\omega}^{*}(\boldsymbol{\xi})$, the uniform exponent of $\boldsymbol{\xi}$ in the sense of dual approximation, is defined as the supremum of such $\gamma$ for which the system of inequalities

$$
\langle\boldsymbol{q} \cdot \boldsymbol{\xi}\rangle \leq t^{-\gamma}, \quad 0<\|\boldsymbol{q}\| \leq t
$$

has an integer solution $\boldsymbol{q}$ for all $t$ large enough. It is clear that always

$$
\hat{\omega}(\boldsymbol{\xi}) \geq 1 / n \text { and } \hat{\omega}^{*}(\boldsymbol{\xi}) \geq n .
$$

In [11] vectors $\boldsymbol{\xi}$ satisfying $\hat{\omega}(\boldsymbol{\xi})>1 / n$ (equivalently, $\hat{\omega}^{*}(\boldsymbol{\xi})>n$ ) were called very singular; clearly very singular implies singular. See [11, Theorem 1.3] for an interpretation of the quantities $\hat{\omega}(\boldsymbol{\xi})$ and $\hat{\omega}^{*}(\boldsymbol{\xi})$ in terms of the rate of divergence of certain trajectories in the space of lattices.

1.2. Theorems of Khintchine and Jarník. Let us say that $\boldsymbol{\xi} \in \mathbb{R}^{n}$ is totally irrational if $1, \xi_{1}, \ldots, \xi_{n}$ are linearly independent over $\mathbb{Q}$. It is easy to see that for not totally irrational vectors $\boldsymbol{\xi}$ one has

$$
\hat{\omega}^{*}(\boldsymbol{\xi})=\infty \quad \text { and } \quad \hat{\omega}(\boldsymbol{\xi}) \geq \frac{1}{n-1} ;
$$

in particular, they clearly are very singular. On the other hand, in [16] Jarník observed that for totally irrational $\boldsymbol{\xi}$ one has the upper bound

$$
\hat{\omega}(\xi) \leq 1 .
$$

In a fundamental paper [19] in the case $n=2$ Khintchine discovered the phenomenon of existence of very singular totally irrational vectors. This was later generalized by Jarník to the setting of systems of linear forms [17]. The following two theorems constitute a special case of Jarník's result.

Theorem A. There exist continuum many totally irrational $\boldsymbol{\xi} \in \mathbb{R}^{n}$ such that $\hat{\omega}^{*}(\boldsymbol{\xi})=\infty$.

Theorem B. There exist continuum many totally irrational $\boldsymbol{\xi} \in \mathbb{R}^{n}$ such that $\hat{\omega}(\boldsymbol{\xi})=1$.

Here we should note that in the case $n=2$ Khintchine deduced Theorem $\mathrm{B}$ from Theorem $\mathrm{A}$ by means of a transference argument. However for $n>2$ Jarník proved Theorem B directly, without using transference. In fact, the transference argument from Jarník's paper [16], which can also be found in the monograph by Cassels [7, Ch. V, §2, Thm. II], when applied to Theorem A gives a weaker conclusion $\hat{\omega}(\boldsymbol{\xi}) \geq \frac{1}{n-1}$.

Further results, generalizations and applications are discussed in Cassels' book [7] and in a survey by the second-named author [27]. We note that Khintchine's method was used by Dani [10] and later by the third-named [35] to exhibit rapidly divergent trajectories of diagonalizable semigroups on homogeneous spaces of higher rank semisimple Lie groups. 
1.3. Approximation on manifolds and fractals. A recurrent theme in Diophantine approximation is the introduction of restrictions on the vector $\boldsymbol{\xi}$, for instance by imposing a functional dependence between its components, or restrictions on the digital expansion of its coefficients. In other terms, one is interested in the Diophantine properties of vectors $\xi$ which are known to lie in a certain subset of $\mathbb{R}^{n}$, such as a fractal or a smooth submanifold. See [3] for history and references, and $[23,22,21$ for developments utilizing dynamics on the space of lattices, and in particular quantitative non-divergence estimates.

As far as singular vectors on fractals or manifolds go, only a few results have been known until recently. Davenport and Schmidt [13, Theorem 3] proved that almost all vectors of the form $\left(x, x^{2}\right)$ are not singular. This was later extended to other manifolds [1, 2, 14, 5]. Recall that a smooth submanifold of $\mathbb{R}^{n}$ is called nondegenerate if at its Lebesgue-almost every point partial derivatives of its parametrizing map up to some order span $\mathbb{R}^{n}$; if $M$ is connected and real analytic, this is equivalent to not lying in any proper affine subspace (we define real analytic manifolds in \$3). Using quantitative non-divergence results obtained in [22], two of the authors in [25, Theorem 1.1] generalized the results of Davenport and Schmidt, proving that the intersection of the set of singular vectors with any smooth nondegenerate manifold has measure zero. They also showed that on a large class of fractal sets, the set of singular vectors has measure zero with respect to the Hausdorff measure on the fractal.

A natural question to ask is whether the above intersection is in fact nontrivial, that is, not contained in the set of totally irrational vectors.

The only examples of curves on which nontrivial singular vectors have been exhibited are rational quadrics in $\mathbb{R}^{2}$ such as the parabola $\left\{\left(x, x^{2}\right): x \in \mathbb{R}\right\}$. This was done by Roy [31, 32]. His result for the parabola was optimal, in the sense that he exhibited the least upper bounds for the sets $\{\hat{\omega}(\boldsymbol{\xi})\}$ and $\left\{\hat{\omega}^{*}(\boldsymbol{\xi})\right\}$ where $\boldsymbol{\xi}$ runs through all totally irrational vectors of the form $\left(x, x^{2}\right)$. Optimal results for quadric hypersurfaces in $\mathbb{R}^{n}$ were very recently obtained by Poëls and Roy [30, 29], complementing upper estimates for uniform Diophantine exponents found earlier by two of the authors [24], see \$1.7.

For a quite general class of higher-dimensional real analytic manifolds this question was addressed in [25, Theorem 1.2]:

Theorem C. Let $\mathcal{S}$ be a connected real analytic submanifold of $\mathbb{R}^{n}$ of dimension at least 2 which is not contained in any proper rational affine subspace of $\mathbb{R}^{n}$. Then there exists a totally irrational singular vector $\boldsymbol{\xi} \in \mathcal{S}$. Moreover, one can find uncountably many such $\boldsymbol{\xi}$ with

$$
\hat{\omega}(\boldsymbol{\xi}) \geq \frac{n^{2}+1}{n\left(n^{2}-1\right)}=\frac{1}{n}+\frac{2}{n\left(n^{2}-1\right)} .
$$

This was actually done in the context of weighted approximation, see \$1.5. The 'moreover' part was not written explicitly in [25], but can be easily derived from [25, Corollary 5.2 and Remark 5.4]. However the proof given in 25] contains a gap, and one of the goals of the present paper is to rectify it by providing a complete proof of a stronger statement. We will discuss the gap in the proof at the end of $\$ 4$. 
1.4. The main result. We now formulate a general result, which extends Theorem $\mathrm{A}$ to quite general subsets $S \subset \mathbb{R}^{n}$, and from which a stronger version of Theorem C follows. The conditions on $S$ will be phrased in terms of its intersections with rational affine hyperplanes. If $\mathbf{m}=\left(m_{0}, m_{1}, \ldots, m_{n}\right) \in$ $\mathbb{Z}^{n+1}$ is a primitive vector, we will denote by $A_{\mathbf{m}}$ the hyperplane

$$
A_{\mathbf{m}} \stackrel{\text { def }}{=}\left\{\xi \in \mathbb{R}^{n}: \sum_{i=1}^{n} m_{i} \xi_{i}=m_{0}\right\} \text {, }
$$

and write

$$
\left|A_{\mathbf{m}}\right| \stackrel{\text { def }}{=}\left\|\left(m_{1}, \ldots, m_{n}\right)\right\| .
$$

We will also work with a generalized version of the uniform exponent for dual approximation. Let $\Phi: \mathbb{Z}^{n} \backslash\{0\} \rightarrow \mathbb{R}_{+}$be a proper function, that is

$$
\text { the set }\left\{\boldsymbol{q} \in \mathbb{Z}^{n}: \Phi(\boldsymbol{q}) \leq C\right\} \text { is finite for any } C>0 .
$$

In accordance with $\Phi$ we define the following irrationality measure function

$$
\psi_{\Phi, \boldsymbol{\xi}}(t) \stackrel{\text { def }}{=} \min _{\boldsymbol{q} \in \mathbb{Z}^{n} \backslash\{\mathbf{0}\}, \Phi(\boldsymbol{q}) \leq t}\langle\boldsymbol{q} \cdot \boldsymbol{\xi}\rangle .
$$

For example for $\Phi(\boldsymbol{q})=\|\boldsymbol{q}\|$ the function $\psi_{\|\cdot\|, \boldsymbol{\xi}}$ can be used to define the uniform exponent of $\boldsymbol{\xi}$ in the sense of dual approximation:

$$
\hat{\omega}^{*}(\boldsymbol{\xi})=\sup \left\{\gamma: \limsup _{t \rightarrow \infty} t^{\gamma} \psi_{\|\cdot\|, \xi}(t)<\infty\right\}
$$

Recall that $S \subset \mathbb{R}^{n}$ is called locally closed if there is an open set $\mathcal{W}$ such that $S=\bar{S} \cap \mathcal{W}$. The following is our main result.

Theorem 1.1. Let $S \subset \mathbb{R}^{n}$ be a nonempty locally closed subset, let $\left\{L_{1}, L_{2}, \ldots\right\}$ and $\left\{L_{1}^{\prime}, L_{2}^{\prime}, \ldots\right\}$ be disjoint collections of distinct closed subsets of $S$, each of which is contained in a rational affine hyperplane in $\mathbb{R}^{n}$, and for each $i$ let $A_{i}$ be a rational affine hyperplane containing $L_{i}$. Assume the following hold:

$$
\bigcup_{i} L_{i} \cup \bigcup_{j} L_{j}^{\prime}=\{x \in S: x \text { is contained in a rational affine hyperplane }\} \text {. }
$$

(b) For each $i$ and each $T>0$,

$$
L_{i}=\overline{\bigcup_{\left|A_{j}\right|>T} L_{i} \cap L_{j}} ;
$$

(c) For each $i$, and for any finite subsets of indices $F, F^{\prime}$ with $i \notin F$, we have

$$
L_{i}=\overline{L_{i} \backslash\left(\bigcup_{k \in F} L_{k} \cup \bigcup_{k^{\prime} \in F^{\prime}} L_{k^{\prime}}^{\prime}\right)} \text {; }
$$

(d) $\bigcup_{i} L_{i}$ is dense in $S$. 
Then for arbitrary $\Phi: \mathbb{Z}^{n} \rightarrow \mathbb{R}_{+}$satisfying (1.8) and for any non-increasing function $\varphi: \mathbb{R}_{+} \rightarrow \mathbb{R}_{+}$, there exist uncountably many totally irrational $\boldsymbol{\xi} \in S$ such that $\psi_{\Phi, \boldsymbol{\xi}}(t) \leq \varphi(t)$ for all large enough $t$.

An application of Theorem 1.1 to $\Phi(\boldsymbol{q})=\|\boldsymbol{q}\|$, in view of (1.10), immediately produces

Corollary 1.2. Let $S \subset \mathbb{R}^{n}$ for which there exist collections $\left\{L_{i}\right\},\left\{L_{j}^{\prime}\right\},\left\{A_{i}\right\}$ satisfying the conditions of Theorem 1.1. Then there exist uncountably many totally irrational $\boldsymbol{\xi} \in S$ such that $\hat{\omega}^{*}(\xi)=\infty$.

From this, a standard transference argument from [16] and [7, Ch. V, §2, Thm. II] readily gives

Corollary 1.3. Let $S$ be as in Corollary 1.2. Then there exist uncountably many totally irrational $\boldsymbol{\xi} \in S$ such that $\hat{\omega}(\boldsymbol{\xi}) \geq \frac{1}{n-1}$.

We note that the above corollary gives a stronger statement than Theorem [C, since the exponent $\frac{n^{2}+1}{n\left(n^{2}-1\right)}=\frac{1}{n-1}-\frac{1}{n(n+1)}$ appearing in (1.5) is strictly smaller than $\frac{1}{n-1}$.

1.5. Approximation with weights. One advantage of the general setup of Theorem 1.1 is the possibility to extend our results to approximation with weights. The weighted setting in Diophantine approximation was initiated by Schmidt [34] and became very popular during recent decades, see e.g. [20]. Consider

$$
\boldsymbol{s}=\left(s_{1}, \ldots, s_{n}\right) \in(0,1)^{n}, \quad s_{1}+\cdots+s_{n}=1,
$$

and put

$$
\rho \stackrel{\text { def }}{=} \max _{1 \leq j \leq n} s_{j}, \quad \delta \stackrel{\text { def }}{=} \min _{1 \leq j \leq n} s_{j} .
$$

Then introduce the $\boldsymbol{s}$-quasinorm $\|\cdot\|_{\boldsymbol{s}}$ on $\mathbb{R}^{n}$ by

$$
\|x\|_{s} \stackrel{\text { def }}{=} \max _{1 \leq j \leq n}\left|x_{j}\right|^{1 / s_{j}}
$$

Clearly $\|\boldsymbol{x}\|_{\boldsymbol{s}}=\|\boldsymbol{x}\|^{n}$ when $\boldsymbol{s}=\left(\frac{1}{n}, \ldots, \frac{1}{n}\right)$. Now we define the weighted uniform exponent $\hat{\omega}_{\boldsymbol{s}}(\boldsymbol{\xi})$ for simultaneous approximation as the supremum of those $\gamma$ for which the system of inequalities

$$
\|\langle q \boldsymbol{\xi}\rangle\|_{\boldsymbol{s}} \leq t^{-n \gamma}, \quad 0<q \leq t
$$

has a solution $q \in \mathbb{Z}_{+}$for all $t$ large enough, and the weighted uniform exponent $\hat{\omega}_{\boldsymbol{s}}^{*}(\boldsymbol{\xi})$ of a linear form $\boldsymbol{\xi}$ as the supremum of those $\gamma$ for which the system of inequalities

$$
\langle\boldsymbol{q} \cdot \boldsymbol{\xi}\rangle \leq t^{-\gamma}, \quad 0<\|\boldsymbol{q}\|_{\boldsymbol{s}} \leq t^{n}
$$

has a solution $\boldsymbol{q} \in \mathbb{Z}^{n}$ for all $t$ large enough. Analogously to (1.3) and (1.4), for totally irrational $\boldsymbol{\xi}$ one always has

$$
\hat{\omega}_{s}^{*}(\xi) \geq n \quad \text { and } \quad \frac{1}{n} \leq \hat{\omega}_{s}(\xi) \leq \frac{1}{\rho n} .
$$

Now, in order to construct vectors with large weighted exponents all one needs is to apply Theorem 1.7 to the function

$$
\Phi_{\boldsymbol{s}}(\boldsymbol{q}) \stackrel{\text { def }}{=}\|\boldsymbol{q}\|_{\boldsymbol{s}}^{1 / n}
$$


observing that one has

$$
\hat{\omega}_{\boldsymbol{s}}^{*}(\boldsymbol{\xi}) \stackrel{\text { def }}{=} \sup \left\{\gamma: \limsup _{t \rightarrow \infty} t^{\gamma} \psi_{\Phi_{\boldsymbol{s}}, \boldsymbol{\xi}}(t)<\infty\right\}
$$

This way we arrive at

Corollary 1.4. Let $S$ be as in Corollary 1.2, and let $\boldsymbol{s}$ be as in (1.13). Then there exist uncountably many totally irrational $\boldsymbol{\xi} \in S$ such that $\hat{\omega}_{\boldsymbol{s}}^{*}(\boldsymbol{\xi})=\infty$.

Exact transference theorems for the weighted setting were obtained quite recently. Improving on a paper by Chow, Ghosh, Guan, Marnat and Simmons [9], German [15] proved a transference inequality which in particular states that

$$
\hat{\omega}_{\boldsymbol{s}}^{*}(\boldsymbol{\xi})=\infty \quad \Longrightarrow \quad \hat{\omega}_{\boldsymbol{s}}^{*}(\boldsymbol{\xi}) \geq \frac{1}{n(1-\delta)},
$$

where $\delta$ is defined in (1.14). This leads to the following

Corollary 1.5. Let $S$ be as in Theorem 1.1, let $s$ be as in (1.13), and let $\delta$ be as in (1.14). Then there exist uncountably many totally irrational $\boldsymbol{\xi} \in S$ such that $\hat{\omega}_{\boldsymbol{s}}(\boldsymbol{\xi}) \geq \frac{1}{n(1-\delta)}$.

1.6. Applications to manifolds and fractals. We now describe two classes of subsets $S \subset \mathbb{R}^{n}$ for which the assumptions of Theorem 1.1 can be verified. The first application involves certain product subsets of $\mathbb{R}^{n}$. Recall that a subset of $\mathbb{R}$ is called perfect if it is compact and has no isolated points.

Theorem 1.6. Let $n \geq 2$ and let $S_{1}, \ldots, S_{n}$ be perfect subsets of $\mathbb{R}$ such that

$\mathbb{Q} \cap S_{k}$ is dense in $S_{k}$ for each $k \in\{1,2\}$.

Let $S=\prod_{j=1}^{n} S_{j}$. Then there are collections $\left\{L_{i}\right\},\left\{L_{j}^{\prime}\right\},\left\{A_{i}\right\}$ satisfying the hypotheses of Theorem 1.1. In particular, the conclusions of Theorem 1.1 and Corollaries 1.2- 1.5 hold for S.

For example, the above theorem applies to products of one-dimensional limit sets of rational iterated function systems such as the middle third Cantor set and its generalizations. Thus as a special case we see that a Cartesian product of two copies of Cantor's middle thirds set contains uncountably many totally irrational singular vectors. The question of determining the Hausdorff dimension of the set of singular vectors in this fractal was raised in the recent paper [6] of Bugeaud, Cheung and Chevallier, and an upper bound was obtained by Khalil [18].

As a second application, let us consider real analytic submanifolds.

Theorem 1.7. Let $\mathcal{S}$ be a connected real analytic submanifold of $\mathbb{R}^{n}$ of dimension at least 2 which is not contained in any proper rational affine subspace of $\mathbb{R}^{n}$. Then there are collections $\left\{L_{i}\right\},\left\{L_{j}^{\prime}\right\},\left\{A_{i}\right\}$ satisfying the hypotheses of Theorem 1.1. In particular, the conclusions of Theorem 1.1] and Corollaries 1.2 1.5 hold for $\mathcal{S}$. 
1.7. Optimality of exponents. One may wonder whether it is possible to strengthen the conclusion of Corollary 1.3 and, for $S$ as in Theorem 1.1, construct totally irrational $\xi \in S$ with $\hat{\omega}(\boldsymbol{\xi})=1$, thereby obtaining an optimal result identical to the conclusion of Theorem B restricted to $S$. However this is not the case. To explain why, we give two examples. First of all we refer to the paper [24], where it is shown that for hypersurfaces of the form

$$
\mathcal{S}=\left\{\xi \in \mathbb{R}^{n}: f(\xi)=1\right\} \subset \mathbb{R}^{n},
$$

where $f$ is a homogeneous polynomial of degree $s$ such that $\#\left\{\boldsymbol{x} \in \mathbb{Q}^{n}: f(\boldsymbol{x})=0\right\}<\infty$, one has

$$
\sup _{\text {totally irrational }} \hat{\boldsymbol{\xi} \in \mathcal{S}}(\boldsymbol{\xi}) \leq H_{n-1, s}
$$

where $H_{n-1, s}<1$ is the unique positive root of the equation $1-x=x \cdot \sum_{k=1}^{d}\left(\frac{x}{s-1}\right)^{k}$. In particular for any totally irrational $\xi$ on the unit sphere

$$
\left\{\left(x_{1}, \ldots, x_{n}\right): \quad x_{1}^{2}+\cdots+x_{n}^{2}=1\right\} \subset \mathbb{R}^{n}
$$

one has $\hat{\omega}(\boldsymbol{\xi}) \leq H_{n-1}$, where $H_{n-1}=H_{n-1,2}$ is the unique positive root of the polynomial $x^{n}+\cdots+x-1$. More general results for quadric hypersurfaces, as well as the optimality of the aforementioned bound, were very recently proved by Poëls and Roy in [30].

In addition to that, in $\$ 5$ below we show that in the case when $\mathcal{S}$ is a so-called badly approximable affine subspace of $\mathbb{R}^{n}$, the value $\hat{\omega}(\boldsymbol{\xi})$ is uniformly bounded away from 1 for any totally irrational $\xi \in \mathcal{S}$.

\section{Proof of Theorem 1.1}

The idea of proof goes back to Khintchine's original argument [19] and has appeared in many incarnations in work on the subject, see [27] for a survey. We retain the notation and assumptions of the theorem; that is,

- $S \subset \mathbb{R}^{n}$ is a nonempty locally closed subset;

- $\mathcal{L} \stackrel{\text { def }}{=}\left\{L_{1}, L_{2}, \ldots\right\}, \mathcal{L}^{\prime} \stackrel{\text { def }}{=}\left\{L_{1}^{\prime}, L_{2}^{\prime}, \ldots\right\}$ are disjoint collections of distinct closed subsets of $S$ such that conditions (a)-(d) of Theorem 1.1 hold;

- $\Phi: \mathbb{Z}^{n} \backslash\{0\} \rightarrow \mathbb{R}_{+}$is such that (1.8) holds;

- $\varphi: \mathbb{R}_{+} \rightarrow \mathbb{R}_{+}$is non-increasing.

Also for a rational affine hyperplane $A_{i}$ as in the statement of the theorem we let $\mathbf{m}_{i} \in \mathbb{Z}^{n+1}$ be a primitive vector so that $A_{i}=A_{\mathbf{m}_{i}}$, where the notation and normalization are as in (1.6).

Proof of Theorem 1.1. Let

$$
\mathcal{B} \stackrel{\text { def }}{=}\left\{\boldsymbol{\xi} \in S: \exists t_{0} \text { such that } \forall t \geq t_{0}, \psi_{\Phi, \boldsymbol{\xi}}(t) \leq \varphi(t) \text { and } \boldsymbol{\xi} \text { is totally irrational }\right\},
$$

and suppose by contradiction that $\mathcal{B}$ is at most countably infinite. Write $\mathcal{B}=\left\{\boldsymbol{b}_{1}, \boldsymbol{b}_{2}, \ldots\right\}$ (in case $\mathcal{B}$ is finite, this is a finite list). Let $\mathcal{W}$ be an open subset of $\mathbb{R}^{n}$ for which $S=\bar{S} \cap \mathcal{W}$. Put $\mathcal{U}_{0}=\mathcal{W}$, 
$\boldsymbol{q}_{0}=0, p_{0}=0, i_{0}=0, \Phi(0)=0$. We will show that for each $\nu \in \mathbb{N}$ there is a bounded open set $\mathcal{U}_{\nu} \subset \mathcal{W}$, and an index $i_{\nu} \in \mathbb{N}$, such that, with the notation

$$
\left(p_{\nu}, \boldsymbol{q}_{\nu}\right) \stackrel{\text { def }}{=} \mathbf{m}_{i_{\nu}}
$$

the following conditions are satisfied:

(1) $\varnothing \neq \overline{S \cap \mathcal{U}_{\nu}} \subset \mathcal{U}_{\nu-1}$;

(2) $i_{\nu}>i_{\nu-1}$ and $\Phi\left(\boldsymbol{q}_{\nu}\right)>\Phi\left(\boldsymbol{q}_{\nu-1}\right)$ for all $\nu \in \mathbb{N}$.

(3) For all $k<\nu, \mathcal{U}_{\nu}$ is disjoint from $L_{k} \cup L_{k}^{\prime} \cup\left\{\boldsymbol{b}_{k}\right\}$.

(4) For all $\nu \in \mathbb{N}$ and all $\xi \in \mathcal{U}_{\nu}$ we have

$$
\left|\boldsymbol{\xi} \cdot \boldsymbol{q}_{\nu-1}-p_{\nu-1}\right|<\varphi\left(\Phi\left(\boldsymbol{q}_{\nu}\right)\right) .
$$

(5) For all $\nu \in \mathbb{N}, \mathcal{U}_{\nu} \cap L_{i_{\nu}} \neq \varnothing$.

To see this suffices, take a point

$$
\xi \in S \cap \bigcap_{\nu} \mathcal{U}_{\nu}=\bigcap_{\nu} \overline{S \cap \mathcal{U}_{\nu}}
$$

This intersection is nonempty since the right-hand side of (2.1) is by (1) an intersection of nonempty nested compact sets, and the equality between both sides of (2.1) follows from the fact that for $\nu \geq 2$, the sets $\overline{\mathcal{U}_{\nu}}$ are contained in $\mathcal{W}$. We will reach a contradiction by showing that both $\boldsymbol{\xi} \notin \mathcal{B}$ and $\boldsymbol{\xi} \in \mathcal{B}$. By $(3), \boldsymbol{\xi}$ is not equal to any of the $\boldsymbol{b}_{i}$ and hence $\boldsymbol{\xi} \notin \mathcal{B}$. Also by $(3), \boldsymbol{\xi}$ is not contained in any of the sets in the collections $\mathcal{L}, \mathcal{L}^{\prime}$, and thus by (1.11), $\boldsymbol{\xi}$ is totally irrational. The function $\varphi$ is nonincreasing by assumption, and so is the irrationality measure function $t \mapsto \psi_{\Phi, \boldsymbol{\xi}}(t)$, as follows from its definition (1.9). The properness condition (1.8) guarantees that $\Phi\left(\boldsymbol{q}_{\nu}\right) \rightarrow \infty$ as $\nu \rightarrow \infty$. By (2), for any $t>t_{0} \stackrel{\text { def }}{=} \Phi\left(\boldsymbol{q}_{1}\right)$ there is $\nu$ with $t \in\left[\Phi\left(\boldsymbol{q}_{\nu}\right), \Phi\left(\boldsymbol{q}_{\nu+1}\right)\right]$ and by (4) we have

$$
\psi_{\Phi, \boldsymbol{\xi}}(t) \leq \psi_{\Phi, \boldsymbol{\xi}}\left(\Phi\left(\boldsymbol{q}_{\nu}\right)\right) \leq\left\langle\boldsymbol{q}_{\nu} \cdot \boldsymbol{\xi}\right\rangle \leq\left|\boldsymbol{q}_{\nu} \cdot \boldsymbol{\xi}-p_{\nu}\right|<\varphi\left(\Phi\left(\boldsymbol{q}_{\nu+1}\right)\right) \leq \varphi(t) .
$$

This shows that $\boldsymbol{\xi} \in \mathcal{B}$.

Note that when utilizing the above properties, we did not require property (5). However we will use it for constructing the sequences $\mathcal{U}_{\nu}, i_{\nu}$.

The inductive construction starts with $\nu=1$. Choose $i_{1} \stackrel{\text { def }}{=} \min \left\{i \in \mathbb{N}: L_{i} \neq \varnothing\right\}$, which exists in view of hypothesis $(\mathrm{d})$, and define $\mathcal{U}_{1}$ to be some open set containing a point in $L_{i_{1}}$ and such that $\overline{\mathcal{U}_{1}} \subset \mathcal{W}$. Then (1) and (5) follow from this choice, and properties (2-4) hold vacuously for $\nu=1$.

Now suppose we have constructed $\mathcal{U}_{k}$ and $i_{k}$ with the required properties for $k=1, \ldots, \nu$, and we explain the construction for $\nu+1$. Let $i=i_{\nu}$. By (5) for $k=\nu$ we have $\mathcal{U}_{\nu} \cap L_{i} \neq \varnothing$. By hypothesis (b) there is an infinite subsequence of indices $j$ such that along this subsequence,

$$
\mathcal{U}_{\nu} \cap L_{i} \cap L_{j} \neq \varnothing \text { and }\left|A_{j}\right| \rightarrow_{j \rightarrow \infty} \infty \text {. }
$$

For each such $j$, write $A_{j}=A_{\mathbf{m}_{j}}, \mathbf{m}_{j}=\left(p_{j}^{\prime}, \boldsymbol{q}_{j}^{\prime}\right)$. Then by (1.7), along this subsequence we have $\left\|\boldsymbol{q}_{j}^{\prime}\right\| \rightarrow \infty$, and hence by the property (1.8) of $\Phi$, we can choose $j>i$ so that $\Phi\left(\boldsymbol{q}_{j}^{\prime}\right)>\Phi\left(\boldsymbol{q}_{\nu}\right)$. We 
then set $i_{\nu+1}=j$. This choice ensures that (2) holds for $\nu+1$. Let

$$
\boldsymbol{\xi}_{1} \in \mathcal{U}_{\nu} \cap L_{i} \cap L_{j}
$$

The point $\boldsymbol{\xi}_{1}$ belongs to $L_{i}$ and hence satisfies $\boldsymbol{\xi}_{1} \cdot \boldsymbol{q}_{\nu}=p_{\nu}$. By continuity, we can take a small neighborhood $\mathcal{V} \subset \mathcal{U}_{\nu}$ around $\boldsymbol{\xi}_{1}$, so that for all $\boldsymbol{\xi} \in \mathcal{V}$ we have

$$
\left|\boldsymbol{\xi} \cdot \boldsymbol{q}_{\nu}-p_{\nu}\right|<\varphi\left(\Phi\left(\boldsymbol{q}_{\nu+1}\right)\right) .
$$

This is the inequality in (4), for $\nu+1$.

Since $\boldsymbol{\xi}_{1} \in L_{j}=L_{i_{\nu+1}}$ we have $\mathcal{V} \cap L_{i_{\nu+1}} \neq \varnothing$, so we can apply hypothesis (c) to find that there is

$$
\boldsymbol{\xi} \in L_{j} \cap \mathcal{V} \backslash \bigcup_{k<\nu+1}\left(L_{k} \cup L_{k}^{\prime} \cup\left\{\boldsymbol{b}_{k}\right\}\right) \text {. }
$$

Furthermore, we can take a small enough neighborhood $\mathcal{U}_{\nu+1}$ of $\boldsymbol{\xi}$ so that

$$
\overline{\mathcal{U}_{\nu+1}} \subset \mathcal{U}_{\nu}, \text { and } \mathcal{U}_{\nu+1} \cap \bigcup_{k<\nu+1}\left(L_{k} \cup L_{k}^{\prime} \cup\left\{\boldsymbol{b}_{k}\right\}\right)=\varnothing
$$

With these choices $\mathcal{U}_{\nu+1}$ and $i_{\nu+1}$ will also satisfy (1), (3) and (5). Thus we have completed the inductive construction.

With Theorem 1.1 in hand, it is easy to complete the

Proof of Theorem 1.6. Recall that we are given $S=\prod_{j=1}^{n} S_{j}$, where $S_{1}, \ldots, S_{n}$ are perfect subsets of $\mathbb{R}$ satisfying (1.15). Let $\mathbf{e}_{1}, \ldots, \mathbf{e}_{n}$ be the standard base vectors, and let $\left\{A_{i}\right\}$ be the collection of all rational hyperplanes which are normal to one of $\mathbf{e}_{1}, \mathbf{e}_{2}$ and have nontrivial intersection with $S$ (where each of the rational hyperplanes appears exactly once). That is, each of the hyperplanes $A_{i}$ is of the form

$$
A_{i}=\left\{\xi \in \mathbb{R}^{n}: \xi_{k_{i}}=\frac{p_{i}}{q_{i}}\right\}, \text { where } p_{i} \in \mathbb{Z}, q_{i} \in \mathbb{N} \text { are coprime, and } k_{i} \in\{1,2\} ;
$$

note that necessarily we have $\frac{p_{i}}{q_{i}} \in S_{k_{i}}$.

For each $i$ define $L_{i} \stackrel{\text { def }}{=} S \cap A_{i}$, and let $\left\{L_{j}^{\prime}\right\}$ denote the collection of non-empty intersections $S \cap A$, where $A$ is a rational affine hyperplane, and the set $L_{j}^{\prime}$ does not appear in the list $\left\{L_{i}\right\}$. We claim that with these choices, hypotheses (a)-(d) of Theorem 1.1 are satisfied.

Indeed, (a) is obvious from the definition, and (d) follows from (1.15). For (b) and (c), suppose for concreteness that $k_{i}=1$. Then it follows from (2.3) that

$$
L_{i}=\left\{\xi \in \mathbb{R}^{n}: \xi_{1}=\frac{p_{i}}{q_{i}} \text { and } \xi_{j} \in S_{j} \forall j \neq 1\right\} .
$$

Let $\boldsymbol{\xi} \in L_{i}$ and let $p_{j} / q_{j}$ be a sequence of distinct rationals in $S_{2}$ satisfying $p_{j} / q_{j} \rightarrow \xi_{2}$. Such a sequence exists since $S_{2}$ is perfect and the rationals are dense in $S_{2}$. Let

$$
L_{j} \stackrel{\text { def }}{=}\left\{\xi \in \mathbb{R}^{n}: \xi_{2}=\frac{p_{j}}{q_{j}}\right\} \cap S
$$

Then it is clear from (2.4) that $L_{i} \cap L_{j}$ contains elements $\boldsymbol{\xi}_{j}$ such that $\boldsymbol{\xi}_{j} \rightarrow \boldsymbol{\xi}$, and such that $\boldsymbol{\xi}_{j}$ differs from $\boldsymbol{\xi}$ only in the 2 nd coordinate. Also $\left|A_{j}\right|=q_{j} \rightarrow \infty$ and so for any $T>0, \xi$ is an accumulation 
point of the sets $L_{i} \cap L_{j}$ with $\left|A_{j}\right|>T$. This proves (b). To show (c), note that because $S_{2}, \ldots, S_{n}$ are perfect, the intersection of the set (2.4) with an arbitrary open subset of $\mathbb{R}^{n}$ cannot lie in a union of finitely many proper affine subspaces of $\mathbb{R}^{n}$ different from $A_{i}$; hence (1.12).

\section{Real anAlytic SUbmanifolds}

Let $k \leq n$, and let $\mathcal{U} \subset \mathbb{R}^{k}$ be open. We say that $f: \mathcal{U} \rightarrow \mathbb{R}^{n}$ is real analytic immersion if it is injective, each of its coordinate functions $f_{i}: \mathcal{U} \rightarrow \mathbb{R}(i=1, \ldots, n)$ is infinitely differentiable, the Taylor series of each $f_{i}$ converges in a neighborhood of every $\boldsymbol{x} \in \mathcal{U}$, and the derivative mapping $d_{x} f: \mathbb{R}^{k} \rightarrow \mathbb{R}^{n}$ has rank $k$. By a $k$-dimensional real analytic submanifold in $\mathbb{R}^{n}$ we mean a subset $\mathcal{M} \subset \mathbb{R}^{n}$ such that for every $\boldsymbol{\xi} \in \mathcal{M}$ there is a neighborhood $\mathcal{V} \subset \mathbb{R}^{n}$ containing $\boldsymbol{\xi}$, an open set $\mathcal{U} \subset \mathbb{R}^{k}$, and a real analytic immersion $f: \mathcal{U} \rightarrow \mathbb{R}^{n}$ such that $\mathcal{V} \cap \mathcal{M}=f(\mathcal{U})$. By a real analytic curve (resp., surface) we mean a connected one-dimensional (resp., two-dimensional) real analytic submanifold. A mapping $h: \mathcal{M} \rightarrow \mathbb{R}^{m}$ is real analytic if for any $\xi, f, \mathcal{U}$ as above, each coordinate function of $h \circ f: \mathcal{U} \rightarrow \mathbb{R}^{m}$ is infinitely differentiable and its Taylor series converges in some neighborhood of $f^{-1}(\xi)$.

The crucial property which distinguishes real analytic submanifolds from smooth manifolds, and follows easily from definitions, is the following. Let $\mathcal{M}_{1}, \mathcal{M}_{2}$ be real analytic submanifolds (where we equip them with the topology inherited from the ambient space $\mathbb{R}^{n}$ ). Then, if the intersection $\mathcal{M}_{1} \cap \mathcal{M}_{2}$ has nonempty interior in $\mathcal{M}_{1}$, then this intersection is open in $\mathcal{M}_{1}$; and thus, if additionally $\mathcal{M}_{1}$ is connected and $\mathcal{M}_{2}$ is closed, then $\mathcal{M}_{1} \subset \mathcal{M}_{2}$.

A subset $\mathcal{N} \subset \mathcal{M}$ is called semianalytic if it is locally described by finitely many equalities and inequalities involving real analytic functions, i.e. for every $\boldsymbol{\xi}_{0} \in \mathcal{N}$ there is an open neighborhood $\mathcal{U}$ containing $\xi_{0}$ such that

$$
\mathcal{N} \cap \mathcal{U}=\left\{\boldsymbol{\xi} \in \mathcal{M} \cap \mathcal{U}: \forall i, h_{i}(\boldsymbol{\xi})=0 \text { and } \forall j, \bar{h}_{j}(\boldsymbol{\xi})>0\right\},
$$

for finitely many real analytic functions $h_{i}, \bar{h}_{j}$ on $\mathcal{M} \cap \mathcal{U}$. For background on the geometry of analytic and semianalytic manifolds we refer the reader to [4] and the references therein. In particular the reader may consult [4] for the definition of the dimension of a semianalytic set.

We will need to decompose semianalytic subsets into analytic submanifolds. In this regard we have the following (see [4, §2]):

Proposition 3.1. Let $\mathcal{N} \subset \mathcal{M}$ be a semianalytic subset of a real analytic submanifold $\mathcal{M} \subset \mathbb{R}^{n}$. Then any connected component of $\mathcal{N}$ is semianalytic, and $\mathcal{N}$ has a locally finite presentation as a disjoint union of sets $\mathcal{N}_{1}, \mathcal{N}_{2}, \ldots$, each of which is a connected analytic submanifold of dimension at most $\operatorname{dim} \mathcal{N}$, and such that

$$
i \neq j, \mathcal{N}_{i} \cap \overline{\mathcal{N}_{j}} \neq \varnothing \Longrightarrow \operatorname{dim} \mathcal{N}_{j}>\operatorname{dim} \mathcal{N}_{i}
$$

It will be easier to work with real analytic surfaces than with manifolds of higher dimension. The reason for this is that in this case it will be possible to describe a stratification as in Proposition 3.1 in topological terms. 
Proposition 3.2. Let $\mathcal{S}$ be a bounded real analytic surface, and let $A$ be an affine hyperplane such that $\mathcal{S} \not \subset A$. Denote by $F$ the set of points $\boldsymbol{\xi} \in \mathcal{S} \cap A$ for which there does not exist a neighborhood $\mathcal{U}$ of $\boldsymbol{\xi}$ such that $\mathcal{U} \cap \mathcal{S} \cap A$ is a real analytic curve. Then $F$ is finite, the number of connected components of $(\mathcal{S} \cap A) \backslash F$ is finite, and each of these connected components is a real analytic curve.

We will refer to the connected components of the set $(\mathcal{S} \cap A) \backslash F$ as the one-dimensional basic components of $\mathcal{S} \cap A$.

Example 3.3. Let $n=3$, let $\mathcal{S}$ be defined by

$$
\mathcal{S}=\{(x, y, x y): x, y \in(-1,1)\},
$$

and let

$$
A=\{(x, y, 0): x, y \in \mathbb{R}\} .
$$

Then $A \cap \mathcal{S}$ is the union of a vertical line $\{x=0\}$ and a horizontal line $\{y=0\}$ in the plane $A$, intersecting at the origin $(0,0,0)$. The set $F$ defined in Proposition 3.2 consists of the origin, and the one-dimensional basic components are four open intervals (two horizontal and two vertical) in $A$.

Proof of Proposition 3.2. Write $\mathcal{S}_{0} \stackrel{\text { def }}{=} \mathcal{S} \cap A$, a semianalytic subset of $\mathcal{S}$. Clearly $\operatorname{dim} \mathcal{S}_{0} \leq 2$ and we claim that $\operatorname{dim} \mathcal{S}_{0} \neq 2$. Indeed, if this were to hold then $\mathcal{S}_{0}$ would be open in $\mathcal{S}$, but also closed since $A$ is a closed subset of $\mathbb{R}^{n}$. By connectedness this would imply $\mathcal{S} \subset A$, contrary to assumption.

Thus $\operatorname{dim} \mathcal{S}_{0} \leq 1$. We treat separately the cases $\operatorname{dim} \mathcal{S}_{0}=0$ and $\operatorname{dim} \mathcal{S}_{0}=1$. If $\mathcal{S}$ has dimension 0 , then each of its connected components is a real analytic submanifold of dimension 0, i.e. $\mathcal{S}_{0}$ is a discrete subset of $\mathcal{S}$. Moreover $\mathcal{S}_{0}$ is finite, since the collection described in Proposition 3.1 is locally finite and $\mathcal{S}$ is bounded, and by definition $F=\mathcal{S}_{0}$.

If $\operatorname{dim} \mathcal{S}_{0}=1$, then by Proposition 3.1 (and using again that $\mathcal{S}$ is bounded) we can write $\mathcal{S}_{0}$ as a disjoint union $F_{0} \cup F_{1}$, where $F_{0}$ is a finite set of points and $F_{1}$ is a finite union of disjoint real analytic curves $\mathcal{N}_{i}$. Such a stratification is not unique, but we choose one so that the cardinality of $F_{0}$ is as small as possible. We claim that with this choice, $F_{0}=F$ and the real analytic curves $\mathcal{N}_{i}$ are the connected components of $\mathcal{S}_{0} \backslash F$.

To see this, note that since the $\mathcal{N}_{i}$ are real analytic curves, any point in any one of the $\mathcal{N}_{i}$ cannot belong to the set $F$, so $F \subset F_{0}$. Suppose if possible that there is some $\xi \in F_{0} \backslash F$. Since $\xi \notin F$, it is not an isolated point of $\mathcal{S}_{0}$. Thus, if we denote by $F_{1}(\xi)$ the collection of curves $\mathcal{N}_{i}$ for which $\boldsymbol{\xi} \in \overline{\mathcal{N}_{i}}$, then $F_{1}(\xi) \neq \varnothing$.

Let $\eta$ be the connected component of $\mathcal{S}_{0} \backslash F$ containing $\xi$. Then $\eta$ is a real analytic curve. By the connectedness of $\eta$ and property (3.1), any $\mathcal{N}_{i}$ in the collection $F_{1}(\boldsymbol{\xi})$ must be contained in $\eta$. Since $\boldsymbol{\xi}$ is a smooth point of $\eta$, i.e. there is a neighborhood $W$ of $\xi$ such that $W \cap \mathcal{S}_{0}=W \cap \eta$, it follows that $F_{1}(\xi)$ consists of two real analytic curves $\mathcal{N}_{i}, \mathcal{N}_{j}$ such that the union $\gamma \stackrel{\text { def }}{=} \mathcal{N}_{i} \cup\{\xi\} \cup \mathcal{N}_{j}$ is also a real analytic curve contained in $\eta$. We can therefore modify $F_{0}$ and $F_{1}$, by replacing $F_{0}, F_{1}$ respectively with

$$
F_{0} \backslash\{\xi\} \quad \text { and } \quad F_{1} \cup\{\xi\}=F_{1} \cup \gamma \backslash\left(\mathcal{N}_{i} \cup \mathcal{N}_{j}\right)
$$


But this contradicts the minimality of $F_{0}$, showing that $F_{0}=F$. Since by (3.1) any boundary point of any $\mathcal{N}_{i}$ is in $F$, the $\mathcal{N}_{i}$ are open and closed as subsets of $\mathcal{S}_{0} \backslash F$. Thus they coincide with the connected components of $\mathcal{S}_{0} \backslash F$.

In order to be in a position to apply Proposition 3.2 we will need the following.

Proposition 3.4. Let $k \geq 2$, and let $\mathcal{M} \subset \mathbb{R}^{n}$ be a connected $k$-dimensional real analytic submanifold which is not contained in a proper rational affine subspace of $\mathbb{R}^{n}$. Then $\mathcal{M}$ contains a bounded real analytic surface which is not contained in a proper rational affine subspace of $\mathbb{R}^{n}$.

Proposition 3.4 is proved by induction on the dimension $k$, the base case $k=2$ being obvious. For $k \geq 3$, the deduction of case $k$ from case $k-1$ follows from the observation that any proper affine rational subspace of $\mathbb{R}^{n}$ is contained in a rational affine hyperplane, and from the following. For each $k$ we denote $I_{k} \stackrel{\text { def }}{=}(0,1)^{k}$ the open unit $k$-dimensional cube.

Lemma 3.5. Suppose that for $k \geq 3, \mathcal{M}$ is a $k$-dimensional real analytic submanifold which is the image of $I_{k}$ under a real analytic immersion $f: I_{k} \rightarrow \mathbb{R}^{n}$. Suppose also that $\mathcal{M}$ is not contained in any rational affine hyperplane. Then there exists $\alpha \in(0,1)$ such that the analytic manifold $f_{\alpha}\left(I_{k-1}\right)$, where

$$
f_{\alpha}: I_{k-1} \rightarrow \mathbb{R}^{n}, \quad f_{\alpha}\left(x_{1}, \ldots, x_{k-1}\right) \stackrel{\text { def }}{=} f\left(x_{1}, \ldots, x_{k-1}, \alpha\right)
$$

does not belong to any rational affine hyperplane.

Proof of Lemma 3.5 (and hence of Proposition 3.4). If the conclusion of the Lemma is not true, then for any $\alpha \in(0,1)$ there exists a rational affine hyperplane $A$ containing the image of the function (3.2). This means that

$$
\bigcup_{\mathbf{m}} f^{-1}\left(A_{\mathbf{m}} \cap \mathcal{M}\right)=I_{k}
$$

where the union is taken over all primitive vectors $\mathbf{m} \in \mathbb{Z}^{n+1}$ and $A_{\mathbf{m}}$ is the rational affine hyperplane defined via (1.6). This is a countable union of closed subsets of $I_{k}$ so by the Baire category theorem, one of them has nonempty interior. That is there is a nonempty open subset $\mathcal{U} \subset I_{k}$ and $\mathbf{m}_{0}$ such that $f(\mathcal{U}) \subset A_{\mathbf{m}_{0}}$. That is, $\mathcal{M} \cap A_{\mathbf{m}_{0}}$ has nonempty interior in $\mathcal{M}$. By analyticity and connectedness of $\mathcal{M}$ we then have $\mathcal{M} \subset A_{\mathbf{m}_{0}}$, contrary to hypothesis.

\section{Proof of Theorem 1.7}

We first explain informally the main difficulty in the proof and the idea that allows us to overcome it. As was mentioned above, the intersections of real analytic submanifolds with affine hyperplanes are semianalytic sets, but they need not themselves be real analytic submanifolds. This makes it tricky to verify the hypotheses of Theorem 1.1. To deal with this, we first pass to the case in which $\mathcal{S}$ is a surface and is not contained in a proper rational affine hyperplane. This means that the intersections $\mathcal{S} \cap A$ can be described by Proposition 3.2, Moreover for some affine hyperplanes $A$, the 
sets $\mathcal{S} \cap A$ can be taken to satisfy a transversality condition which implies that they are real analytic curves. Specifically in the proof below, the $L_{i}$ will be closed real analytic curves, while the $L_{j}^{\prime}$ will be basic one-dimensional components of one-dimensional semianalytic sets. We now proceed to the details of the argument.

Proof of Theorem 1.7. By Proposition 3.4 we can assume that $\mathcal{S}$ is bounded, connected and twodimensional. Let $\left\{A_{i}\right\}$ be the collection as in the proof of Theorem 1.6, that is the $A_{i}$ are the affine rational hyperplanes normal to one of the two standard basis vectors $\mathbf{e}_{1}, \mathbf{e}_{2}$. For each $\boldsymbol{\xi} \in \mathcal{S}$, the tangent space $T_{\xi} \mathcal{S}$ is a two dimensional affine subspace of $\mathbb{R}^{n}$ passing through $\boldsymbol{\xi}$. Recall that $\mathcal{S}$ is not contained in any proper rational affine subspace of $\mathbb{R}^{n}$; thus, by possibly replacing $\mathcal{S}$ with its smaller connected open subset, we can assume that for every $\boldsymbol{\xi} \in \mathcal{S}$, the tangent space $T_{\boldsymbol{\xi}} \mathcal{S}$ is not normal to either of $\mathbf{e}_{1}, \mathbf{e}_{2}$. This implies that we can view $\mathcal{S}$ as a graph of a smooth function over its projection to the two-dimensional space $V_{12} \stackrel{\text { def }}{=} \operatorname{span}\left(\mathbf{e}_{1}, \mathbf{e}_{2}\right) \cong \mathbb{R}^{2}$. This implies furthermore that for each $i$ and each $\boldsymbol{\xi} \in \mathcal{S} \cap A_{i}$, the intersection $T_{\xi} \mathcal{S} \cap A_{i}$ is a transversal intersection, that is, an affine subspace of dimension one. By taking $\mathcal{S}$ smaller, we can ensure that its projection to the plane $V_{12}$ is an open bounded convex set. Now define $L_{i} \stackrel{\text { def }}{=} S \cap A_{i}$ (where we only take those indices $i$ for which $L_{i}$ is not empty). Each $L_{i}$ is closed as a subset of $\mathcal{S}$, and by the implicit function theorem it is a real analytic curve. Since the projection of $\mathcal{S}$ on $V_{12}$ is convex, each $L_{i}$ is also connected.

Having defined the collections $\left\{L_{i}\right\},\left\{A_{i}\right\}$ we now define the collection $\left\{L_{j}^{\prime}\right\}$. For any rational affine hyperplane $A$ for which $\mathcal{S} \cap A$ is nonempty, we have by Proposition 3.2 its one-dimensional basic components. There are at most countably many such sets $\left\{\gamma_{j}\right\}$ where each $\gamma_{j}$ is a real analytic curve whose closure $\overline{\gamma_{j}}$ satisfies that $\overline{\gamma_{j}} \backslash \gamma_{j}$ consists of at most two points. We take

$$
\left\{L_{j}^{\prime}\right\} \stackrel{\text { def }}{=}\left\{\overline{\gamma_{j}}: \forall i, \gamma_{j} \not \subset L_{i}\right\}
$$

We claim that with these choices, conditions $(\mathrm{a})-(\mathrm{d})$ of Theorem 1.1 are satisfied (note that as a real analytic submanifold of $\mathbb{R}^{n}, \mathcal{S}$ is locally closed).

Properties (a), (b), (d) are straightforward. Indeed, since each $L_{i}$ is a connected real analytic curve, the condition $\gamma_{j} \not \subset L_{i}$ is equivalent to $\overline{\gamma_{j}} \not \subset L_{i}$. Also the sets $\overline{\gamma_{j}}$ contain all points of $\mathcal{S}$ which belong to rational affine hyperplanes but not to one of the hyperplanes $A_{i}$. Thus we have (a). For (d) note we can apply the projection to the plane $V_{12}$, since $\mathcal{S}$ is a graph over this plane. By construction, the projections of the $L_{i}$ form a dense collection of horizontal lines and a dense collection of vertical lines. In particular (d) holds. For (b) we continue to work in the plane $V_{12}$. For every point $\boldsymbol{\xi}$ on (say) a horizontal line $\ell \subset V_{12}$, which is the projection of some $L_{i}$, there is a sequence of intersection points $\boldsymbol{\xi}_{j}$ of $\ell$ with vertical lines such that $\boldsymbol{\xi}_{j} \rightarrow \boldsymbol{\xi}$ and $\boldsymbol{\xi}_{j}$ is contained in spaces $A_{j}$. A computation similar to the one used in the proof of Theorem 1.6 shows that along this sequence, we have $\left|A_{j}\right| \rightarrow \infty$, and (b) follows.

For (c) we argue as follows. Let $F, F^{\prime}$ be as in statement (c). The set $L_{i}$ is a real analytic curve and for each $k \in F, L_{i} \cap L_{k}$ is either empty or consists of a single point. Now let $k^{\prime} \in F^{\prime}$, and suppose by contradiction that $L_{k^{\prime}}^{\prime} \cap L_{i}$ has nonempty interior, relative to the topology on $L_{i}$. Then, since $L_{k^{\prime}}^{\prime}$ 
is the closure of a real analytic curve $\gamma$ with $\bar{\gamma} \backslash \gamma$ consisting of at most two points, $L_{i} \cap \gamma$ also has nonempty interior relative to $L_{i}$, and, since the dimensions are both equal to one, $\gamma \cap L_{i}$ also has nonempty interior relative to the topology of $\gamma$. Since $L_{i}$ is closed and $\gamma$ is connected, this means that $\gamma \subset L_{i}$, contradicting the definition of $L_{k^{\prime}}^{\prime}$.

We close the section by commenting on Theorem C and its proof given in [25], which, as was mentioned in the introduction, contained an error. Since in this paper we prove a strengthening, namely Theorem [1.7, we do not rewrite the proof of [25] completely. Rather we explain the gap in the proof and sketch how it can be fixed.

Theorem $\mathrm{C}$ is derived in [25] from an abstract result [25, Theorem 5.1], which is similar to Theorem 1.1 (abstracting Khintchine's classical argument). The statement of [25, Theorem 5.1] involves two countable lists $X_{1}, X_{2}, \ldots$ and $X_{1}^{\prime}, X_{2}^{\prime}, \ldots$ of closed subsets of a subset $X$ of a Lie group. The application deals with a real analytic submanifold $\mathcal{S} \subset \mathbb{R}^{n}$ of dimension at least two, embedded in the group $\mathrm{SL}_{n+1}(\mathbb{R})$. In order to conclude that $\mathcal{S}$ contains totally irrational singular vectors $\boldsymbol{\xi}$, and to obtain a bound on their associated parameter $\hat{\omega}(\boldsymbol{\xi})$, some conditions on the sets $X_{i}, X_{j}^{\prime}$ must be checked. One of these is the following transversality condition:

$$
\text { for every } i, j, X_{i}=\overline{X_{i} \backslash X_{j}^{\prime}}
$$

(which is analogous to hypothesis (c) of Theorem 1.1). The argument given in [25] defines the $X_{i}, X_{j}^{\prime}$ as connected components of the intersection of $\mathcal{S}$ with rational affine hyperplanes. It is then erroneously claimed that (4.1) holds for these choices. Indeed, with the notations of Example 3.3, set $A_{1} \stackrel{\text { def }}{=} A$ and $A_{1}^{\prime} \stackrel{\text { def }}{=}\{(0, y, z): y, z \in \mathbb{R}\}$. Then $X_{1}=\mathcal{S} \cap A_{1}$ is the union of two lines intersecting at a point, and $X_{1}^{\prime}=\mathcal{S} \cap A_{1}^{\prime}$ is one of these lines. So (4.1) fails.

It is possible to rectify the proof by adapting some of the arguments we used in the proof of Theorem 1.7; namely, by replacing $\mathcal{S}$ with a two-dimensional real analytic submanifold, and adjusting the definitions of the sets $X_{i}, X_{i}^{\prime}$ using the notion of basic components. We leave the details to the reader.

\section{BADly APPROXIMABle SUBSPACES}

In this section we give upper bounds for the exponent $\hat{\omega}(\boldsymbol{\xi})$ for points $\boldsymbol{\xi} \in A$ in case when an $s$ dimensional affine subspace $A$ of $\mathbb{R}^{n}$ is badly approximable. To define the latter property, we identify $\mathbb{R}^{n}$ with the affine subspace

$$
\mathbb{R}_{1}^{n}:=\left\{\mathbf{x}=\left(x_{0}, x_{1}, \ldots, x_{n}\right) \in \mathbb{R}^{n+1}: x_{0}=1\right\}
$$

and consider the affine subspace

$$
\mathcal{A} \stackrel{\text { def }}{=}\left\{\mathbf{x} \in \mathbb{R}^{n+1}: x_{0}=1,\left(x_{1}, \ldots, x_{n}\right) \in A\right\} .
$$

Let us define the linear subspace

$$
L_{A} \stackrel{\text { def }}{=} \operatorname{span} \mathcal{A} \subset \mathbb{R}^{n+1} \text {. }
$$


It is clear that $L_{A}$ has dimension $s+1$. From Minkowski's convex body theorem it follows that there exists a constant $C$ dependent only on $n$ such that for any $A$ there exists infinitely many integer vectors $\mathbf{m} \in \mathbb{Z}^{n+1}$ such that

$$
\operatorname{dist}\left(L_{A}, \mathbf{m}\right)^{n-s} \cdot\|\mathbf{m}\|^{s+1}<C .
$$

We define $A$ to be a badly approximable subspace if

$$
\inf _{\mathbf{m} \in \mathbb{Z}^{n+1} \backslash\{0\}} \operatorname{dist}\left(L_{A}, \mathbf{m}\right)^{n-s} \cdot\|\mathbf{m}\|^{s+1}>0 .
$$

It is clear that badly approximable subspaces exist. Moreover from a famous theorem of Schmidt [33] it follows that they form a thick set (that is, the set of badly approximable subspaces in any nonempty open subset of the Grassmanian of all $s$-dimensional affine subspaces of $\mathbb{R}^{n}$ has full Hausdorff dimension). Indeed, without loss of generality one can parametrize $A$ in the following form:

$$
A=\left\{\xi=\left(\begin{array}{c}
x \\
y_{0}+Y x
\end{array}\right): x \in \mathbb{R}^{s}\right\}
$$

where $Y \in M_{n-s, s}$ and $\boldsymbol{y}_{0} \in \mathbb{R}^{n-s}$. Define

$$
w_{s, n} \stackrel{\text { def }}{=} \frac{s+1}{n-s} .
$$

Then it is easy to see that $A$ is badly approximable if and only if the augmented matrix

$$
\widetilde{Y}:=\left[\begin{array}{ll}
\boldsymbol{y}_{0} & Y
\end{array}\right] \in M_{n-s, s+1}
$$

is badly approximable, that is, if

$$
\inf _{\mathbf{q} \in \mathbb{Z}^{s+1} \backslash\{0\}}\|\mathbf{q}\|^{w_{s, n}}\|\langle\widetilde{Y} \mathbf{q}\rangle\|>0
$$

Note that

$$
w_{s, n}<1 \quad \Longleftrightarrow \quad s<\frac{n-1}{2} .
$$

Let $W_{s, n}$ be the unique root of the equation

$$
x^{n+1}-w_{s, n}^{n-1}\left(1+w_{s, n}\right) x+w_{s, n}^{n}=0
$$

in the interval $\left(0, w_{s, n}\right)$.

Proposition 5.1. Let $A$ be an s-dimensional badly approximable affine subspace of $\mathbb{R}^{n}$. Then:

(i) for any $\boldsymbol{\xi} \in A$ one has

$$
\hat{\omega}(\boldsymbol{\xi}) \leq w_{s, n}
$$

(ii) for any totally irrational $\boldsymbol{\xi} \in A$ one has

$$
\hat{\omega}(\boldsymbol{\xi}) \leq W_{s, n} .
$$

Remark 5.2. In view of (5.3), when $\boldsymbol{\xi}$ is totally irrational, the estimate in (i) is non-trivial only if $s<\frac{n-1}{2}$. If $s$ is fixed, then $w_{s, n}=O\left(\frac{1}{n}\right)$ as $n \rightarrow \infty$, which shows that for large $n$ the conclusion of Corollary 1.3, that is, the existence of uncountably many totally irrational $\boldsymbol{\xi} \in S$ with $\hat{\omega}(\boldsymbol{\xi}) \geq \frac{1}{n-1}$, is close to optimal, in some sense. Statement (ii) gives a slight improvement of this bound. 
In particular, we can consider the following examples:

1) if $n=4$ and $s=1$, the inequality (5.5) gives $\hat{\omega} \leq w_{1,4}=\frac{2}{3}$;

2) if $n=2$ and $s=1$, we have $w_{1,2}=2$. Equation (5.4) is now $x^{3}-6 x+4=0$, and the inequality (5.6) gives

$$
\hat{\omega} \leq W_{1,2}=\sqrt{3}-1=0.732 \ldots
$$

3 ) if $n=3$ and $s=1$, we have $w_{1,3}=1$, equation (5.4) has the form $x^{4}-2 x+1=0$, and the inequality (5.6) gives

$$
\hat{\omega} \leq W_{1,3}=0.54 \ldots
$$

4) if $n=3$ and $s=2$, we have $w_{2,3}=3$, equation (5.4) is now $x^{3}-36 x+27=0$, and the inequality (5.6) gives

$$
\hat{\omega} \leq W_{2,3}=0.759 \ldots
$$

Proof of Proposition 5.1. To prove (i), we will use the following elementary

Lemma 5.3. If $\boldsymbol{\xi} \in A$ and $A$ is badly approximable, then there exists a positive $c$ such that for every $q \in \mathbb{N}$ we have

$$
\|\langle q \boldsymbol{\xi}\rangle\| \geq c q^{-w_{n, s}}
$$

From this lemma (5.5) follows immediately.

Proof of Lemma 5.3. We will use the parameterization (5.1). Assume the contrary, that is, for some $\boldsymbol{\xi}=\left(\begin{array}{c}\boldsymbol{x} \\ \boldsymbol{y}_{0}+Y \boldsymbol{x}\end{array}\right)$ and any $\varepsilon>0$ there exists $\boldsymbol{m}=\left(\begin{array}{l}\boldsymbol{p} \\ \boldsymbol{r}\end{array}\right) \in \mathbb{Z}^{n}$ such that

$$
\|q \boldsymbol{\xi}-\boldsymbol{m}\|=\left\|\left(\begin{array}{c}
q \boldsymbol{x} \\
q \boldsymbol{y}_{0}+Y(q \boldsymbol{x})
\end{array}\right)-\left(\begin{array}{l}
\boldsymbol{p} \\
\boldsymbol{r}
\end{array}\right)\right\|<\varepsilon q^{-w_{n, s}} .
$$

In particular, $\|q \boldsymbol{x}-\boldsymbol{p}\|<\varepsilon q^{-w_{n, s}}$; thus, if we define $\mathbf{q}:=\left(\begin{array}{c}q \\ \boldsymbol{p}\end{array}\right)$, it follows that

$$
\|\mathbf{q}\| \leq C q \quad \text { for some } C=C(\boldsymbol{x}) \text { independent on } q, \boldsymbol{p} \text { and } \varepsilon \text {. }
$$

Note that

$$
q \boldsymbol{y}_{0}+Y(q \boldsymbol{x})-\boldsymbol{r}=q \boldsymbol{y}_{0}+Y(\boldsymbol{p}+q \boldsymbol{x}-\boldsymbol{p})-\boldsymbol{r}=\widetilde{Y} \mathbf{q}-\boldsymbol{r}+Y(q \boldsymbol{x}-\boldsymbol{p}),
$$

where $\tilde{Y}$ is as in (5.2). Hence

$$
\|\langle\widetilde{Y} \mathbf{q}\rangle\| \leq\left\|q \boldsymbol{y}_{0}+Y(q \boldsymbol{x})-\boldsymbol{r}\right\|+\|Y(q \boldsymbol{x}-\boldsymbol{p})\|<\widetilde{C} \varepsilon q^{-w_{n, s}},
$$

where $\widetilde{C}$ is a constant depending only on $Y$. Since $\varepsilon$ was arbitrary and in view of (5.8), this shows that $\widetilde{Y}$, and hence the subspace $A$, is not badly approximable.

To prove (ii) we consider the ordinary Diophantine exponent $\omega=\omega(\boldsymbol{\xi})$, defined as supremum of those $\gamma>0$ for which the inequality

$$
\|\langle q \boldsymbol{\xi}\rangle\|<q^{-\gamma}
$$


has infinitely many solutions in $q \in \mathbb{N}$. It is clear from (5.7) that for $\xi \in A$ one has

$$
\omega(\boldsymbol{\xi}) \leq \frac{s+1}{n-s}=w_{s, n}
$$

Then we apply the inequality

$$
\frac{\omega(\boldsymbol{\xi})}{\hat{\omega}(\boldsymbol{\xi})} \geq G_{n}
$$

where $G_{n}$ the unique positive root of the equation

$$
x^{n-1}=\frac{\hat{\omega}}{1-\hat{\omega}}\left(x^{n-2}+x^{n-3}+\cdots+x+1\right),
$$

which is valid for all totally irrational $\xi \in \mathbb{R}^{n}$. This result was proven in [26], and a short and beautiful proof was given recently in 28].

Note that as $\frac{1}{n} \leq \hat{\omega} \leq 1$, we have $G_{n} \geq 1$ and $G_{n}=1$ if and only if $\hat{\omega}=\frac{1}{n}$. We see that $G_{n}$ is also a root of the simpler equation

$$
(1-\hat{\omega}) x+\frac{\hat{\omega}}{x^{n-1}}=1 \Longleftrightarrow g(x) \stackrel{\text { def }}{=}(1-\hat{\omega}) x^{n}-x^{n-1}+\hat{\omega}=0 .
$$

The polynomial $g(x)$ in the interval $x \geq 1$ has the unique root $G_{n}$. Hence

$$
x \geq G_{n} \Longleftrightarrow(1-\hat{\omega}) x^{n}-x^{n-1}+\hat{\omega} \geq 0 .
$$

Moreover, since

$$
\max _{\frac{1}{n} \leq z \leq 1}\left(z(1-z)^{n-1}\right)=\frac{1}{n} \cdot\left(1-\frac{1}{n}\right)^{n-1}
$$

we have

$$
\hat{\omega}(1-\hat{\omega})^{n-1} \leq \frac{1}{n} \cdot\left(1-\frac{1}{n}\right)^{n-1} .
$$

Therefore $g\left(\frac{1}{1-\hat{\omega}} \cdot \frac{n-1}{n}\right) \leq 0$, and we deduce that $G_{n} \geq \frac{1}{1-\hat{\omega}} \cdot \frac{n-1}{n}$.

Now (5.10) leads to

$$
(1-\hat{\omega}) \omega^{n}-\omega^{n-1} \hat{\omega}+\hat{\omega}^{n+1} \geq 0 .
$$

The function

$$
f(y) \stackrel{\text { def }}{=}(1-\hat{\omega}) y^{n}-y^{n-1} \hat{\omega}+\hat{\omega}^{n+1}
$$

increases when $y \geq \frac{\hat{\omega}}{1-\hat{\omega}} \cdot \frac{n-1}{n}$. Since $\omega \geq \hat{\omega} \cdot G_{n} \geq \frac{\hat{\omega}}{1-\hat{\omega}} \cdot \frac{n-1}{n}$, we deduce from (5.9) the inequality

$$
(1-\hat{\omega}) w_{s, n}^{n}-w_{s, n}^{n-1} \hat{\omega}+\hat{\omega}^{n+1} \geq 0
$$

which immediately gives (5.6).

Acknowledgements. The authors were supported by NSF grant DMS-1600814, RFBR grant No. 1801-00886 and BSF grant 2016256, respectively. This work was started during the second-named author's visit to Brandeis University, whose hospitality is gratefully acknowledged. The authors are also grateful to the MATRIX institute for providing a stimulating atmosphere for a productive collaboration, and to the anonymous referee for taking a close look at the paper and catching some errors. 


\section{REFERENCES}

[1] R. C. Baker, Metric diophantine approximation on manifolds, J. Lond. Math. Soc. (2) 14 (1976), 43-48.

[2] _ Dirichlet's theorem on diophantine approximation, Math. Proc. Cambridge Phil. Soc. 83 (1978), 37-59.

[3] V. I. Bernik and M. M. Dodson, Metric Diophantine approximation on manifolds, volume 137 of Cambridge Tracts in Mathematics, Cambridge University Press, Cambridge, 1999.

[4] E. Bierstone and P. D. Milman, Semianalytic and subanalytic sets, Inst. Hautes Études Sci. Publ. Math. 67 (1988), 5-42.

[5] Y. Bugeaud, Approximation by algebraic integers and Hausdorff dimension, J. London Math. Soc. (2) 65 (2002), no. 3, 547-559.

[6] Y. Bugeaud, Y. Cheung, and N. Chevallier, Hausdorff dimension and uniform exponents in dimension two, Math. Proc. Cambridge Phil. Soc. 167 (2018), no. 2, 249-284.

[7] J.W.S. Cassels, An Introduction to Diophantine Approximation, Cambridge Tracts in Math. and Math. Phys. 45, Cambridge University Press, Cambridge, 1957.

[8] Y. Cheung and N. Chevallier, Hausdorff dimension of singular vectors, Duke Math. J. 165 (2016), no. 12, 2273-2329.

[9] S. Chow, A. Ghosh, L. Guan, A. Marnat, and D. Simmons, Diophantine transference inequalities: weighted, inhomogeneous, and intermediate exponents, preprint available at arXiv:1808.07184 (2018).

[10] S. G. Dani, Divergent trajectories of flows on homogeneous spaces and Diophantine approximation, J. Reine Angew. Math. 359 (1985), 55-89.

[11] T. Das, L. Fishman, D. Simmons, and M. Urbański, A variational principle in the parametric geometry of numbers, preprint available at arXiv:1901.06602 (2019).

[12] H. Davenport and W. M. Schmidt, Approximation to real numbers by algebraic integers, Acta Arith. 15 (1969), 393-416.

[13] _ Dirichlet's theorem on diophantine approximation, in: Symposia Mathematica, Vol. IV (INDAM, Rome, 1968/69), 1970, pp. 113-132.

[14] M. Dodson, B. Rynne, and J. Vickers, Dirichlet's theorem and Diophantine approximation on manifolds, J. Number Theory 36 (1990), no. 1, 85-88.

[15] O. N. German, Transference theorems for Diophantine approximation with weights, preprint available at arXiv:1905.01512 (2019).

[16] V. Jarník, Zum Khintchineschen «Übertragungssatz», Trav. Inst. Math. Tbilissi 3 (1938), 193-212.

[17] _ Eine Bemerkung über diophantische Approximationen, Math. Z. 72, no. 1 (1959), $187-191$.

[18] O. Khalil, Singular vectors on fractals and projections of self-similar measures, preprint available at arxiv.org/abs/1904.11330 (2019). 
[19] A. Ya. Khintchine, Über eine Klasse linearer Diophantischer Approximationen, Rend. Circ. Math. Palermo 50 (1926), 170-195.

[20] D. Kleinbock, Flows on homogeneous spaces and Diophantine properties of matrices, Duke Math. J. 95 (1998), no. 1, 107-124.

[21] _ Quantitative nondivergence and its Diophantine applications, in: Homogeneous flows, moduli spaces and arithmetic, pages 131-153, Clay Math. Proc., 10, Amer. Math. Soc., Providence, RI, 2010.

[22] D. Kleinbock, E. Lindenstrauss, and B. Weiss, On fractal measures and diophantine approximation, Selecta Math. (N.S.) 10 (2004), no. 4, 479-523.

[23] D. Kleinbock and G. A. Margulis, Flows on homogeneous spaces and Diophantine approximation on manifolds, Ann. of Math. (2), 148 (1998), no. 1, 339-360.

[24] D. Kleinbock and N. Moshchevitin, Simultaneous Diophantine approximation: sums of squares and homogeneous polynomials, Acta Arith. 190 (2019), 87-100.

[25] D. Kleinbock and B. Weiss, Friendly measures, homogeneous flows and singular vectors, in: Algebraic and Topological Dynamics, Contemp. Math. 211, Amer. Math. Soc., Providence, RI, 2005, pp. 281-292.

[26] A. Marnat and N. Moshchevitin, An optimal bound for the ratio between ordinary and uniform exponents of Diophantine approximation, preprint available at arXiv:1802.03081v3 (2018).

[27] N. Moshchevitin, Khintchine's singular Diophantine systems and their applications, Russian Math. Surveys 65 (2010), no. 3, 433-511.

[28] V. Nguyen, A. Poëls and D. Roy, A transference principle for simultaneous rational approximation, preprint available at arXiv:1908.11777 (2019).

[29] A. Poëls, A class of maximally singular sets for rational approximation, preprint available at arXiv:1909.12159 (2019).

[30] A. Poëls and D. Roy Rational approximation to real points on quadratic hypersurfarces, preprint available at arXiv:1909.01499 (2019).

[31] D. Roy, Approximation simultanée d'un nombre et de son carré, C. R. Math. Acad. Sci. Paris 336 (2003), no. 1, 1-6.

[32] _ - On Two Exponents of Approximation Related to a Real Number and Its Square, Canad. J. Math. 59 (2007), no. 1, 211-224.

[33] W. M. Schmidt, Badly approximable systems of linear forms, J. Number Theory 1 (1969), 139-154.

[34] _ Open problems in Diophantine Approximations, in: Approximations Diophantiennes et nombres transcendants, Luminy, 1982, Progress in Mathematics, Birkhäuser, 271289 (1983).

[35] B. Weiss, Divergent trajectories on noncompact parameter spaces, Geom. Funct. Anal. 14 (2004), no. 1, 94-149. 
Department of Mathematics, Brandeis University, Waltham MA, USA 02454-9110

E-mail address: kleinboc@brandeis.edu

Moscow State University, Leninskie Gory 1, Moscow, Russia, 1119991, And Astrakhan State University, Tatishcheva 20A, Astrakhan, Russia, 414056

E-mail address: moshchevitin@gmail.com

Department of Mathematics, Tel Aviv University, Tel Aviv, Israel

E-mail address: barakw@post.tau.ac.il 